\title{
Beam dynamics in transverse deflecting rf structures
}

\author{
Klaus Floettmann \\ DESY, Notkestrasse 85, 22603 Hamburg, Germany \\ Valentin V. Paramonov \\ Institute for Nuclear Research, 117312 Moscow, Russia \\ (Received 19 August 2013; published 5 February 2014)
}

\begin{abstract}
The beam dynamics in transverse deflecting structures, operating in streaking mode, is discussed concentrating on slightly nonrelativistic particle energies. Transverse offsets of the average trajectory, bunch lengthening, and defocusing as well as emittance growth due to nonlinearities of the cavity field are considered. The analysis of the deflecting field reveals the origin of nonlinearities and leads to proposals for their suppression. An optimized cavity design which combines minimal aberrations with a high rf efficiency is proposed.
\end{abstract}

DOI: 10.1103/PhysRevSTAB.17.024001

PACS numbers: 29.27.-a, 41.85.-p, 07.78.+s

\section{INTRODUCTION}

Transverse deflecting structures (TDS) have become widely utilized diagnostics components for investigations of the longitudinal phase space in high brightness beams applications [1,2]. Conceptually equal to an inline streak camera, a TDS shears a bunch of charged particles in the transverse direction, i.e., it introduces a correlation of a transverse momentum to the longitudinal position in the bunch. This correlation can be used to image the longitudinal particle distribution with appropriate beam optics. An intriguing concept is to use a TDS in electron diffraction experiments for single-shot time-resolved measurements $[3,4]$. The diffraction pattern, rather than being a pattern of spots, becomes a pattern of stripes in this case and temporal variations in the structure of the target material could be seen as variation of the position, width and strength of the stripes.

Electron diffractometer operate at low energies, often below $100 \mathrm{keV}$, but the increasing need for even higher performance has led also to the development of facilities operating in the range of a few $\mathrm{MeV}$ where space charge effects are considerably reduced, as for example the REGAE facility [5]. This energy is however still small as compared to other accelerators which often make use of transverse deflecting structures, e.g., free-electron laser facilities. In addition, even more demanding transverse emittance requirements need to be met for electron diffraction experiments than for free-electron lasers. For high quality diffraction patterns normalized emittances $<50 \mathrm{~nm}$ are required and have been demonstrated at charges $<1 \mathrm{pC}[6]$.

\footnotetext{
*Klaus.Floettmann@DESY.De

Published by the American Physical Society under the terms of the Creative Commons Attribution 3.0 License. Further distribution of this work must maintain attribution to the author(s) and the published articles title, journal citation, and DOI.
}

The following discussion of the beam dynamics in transverse deflecting structures concentrates thus on two aspects, i.e., the operation at a few $\mathrm{MeV}$ energies and on emittance degradations which limit the resolution achievable with a TDS.

\section{ELECTRON DIFFRACTION WITH A TRANSVERSE DEFLECTING STRUCTURE}

From an electron beam optics point of view, electron diffraction in a target material and the action of a transverse deflecting structure onto the beam omits a comparable problem. In both cases, the beam or a part of the beam receives a transverse momentum which needs to be measured by an appropriate imaging optics and a screen. In order to maximize the effect onto the beam, the intrinsic divergence of the beam at the location of the interaction, i.e., the target or the TDS, should be minimized and thus the beam size should be large. The imaging optics transfers the induced momentum into a measurable distance on the screen. The ratio between this distance and the natural beam size at the location of the screen should be maximized, which can be realized by focusing the beam to a small spot, such that the phase advance between target or TDS and screen is 90 degrees. Another approach is to let the beam drift for a long enough distance so that the phase advance becomes 90 degrees. In the first case a small image is realized in a short distance, while in the second case a large image is realized and a longer distance is needed.

For an imaging optics with a 90 degree phase advance the resolution of the measurement is given as

$$
R=\frac{\epsilon}{\sigma_{x} S} .
$$

In the case of a diffraction pattern $R$ describes the ratio between the size of the central spot to the distance of a 
diffraction spot (relative to the central beam), while it describes the ratio of the size of an unstreaked beam to the size of a streaked beam of unit length in the case of the TDS. In the first case $\sigma_{x}$ is the beam size on the target and $S$ is the diffraction angle, while $\sigma_{x}$ is the beam size in the TDS and the shear parameter $S=\frac{e k V(\varphi)}{c p_{z}}$ describes the angle per unit length introduced by the TDS in the second case. Here $p_{z}$ is the longitudinal momentum of the beam, $V(\varphi)$ and $k$ are the deflecting voltage and wave number of the $\mathrm{rf}$ deflector, $e$ and $c$ are elementary charge and speed of light, and $\epsilon$ is the geometrical beam emittance. (The voltage depends on the phase $\varphi$ at which the structure is traversed.) The product of the spot size $\sigma_{x}$ with the angle $S$ (in case of the TDS multiplied with the length of the bunch) has the unit of an emittance, $R$ can hence be understood as the ratio of the beam emittance to an induced emittance. Both emittances scale inversely to the beam momentum so that $R$ is independent of the beam energy.

Small transverse emittances as in diffraction experiments allow for a high temporal resolution power $R^{-1}$ at moderate deflecting voltage provided the emittance is maintained while traversing the TDS. The beam dynamics in TDSs is hence the main subject of the discussion following below after some general considerations.

The small emittances required for high quality diffraction experiments can only be achieved at low bunch charges. On the other hand, a large number of electrons is desirable in order to reduce statistical fluctuations and to increase the signal-to-noise ratio. Even with advanced detectors allowing for single electron detection, $10^{5}$ electrons are required to generate a high quality diffraction pattern [7]. If a TDS is applied a long bunch with higher total charge will be employed than in a pump-probe type experiment. The charge will however be distributed over a larger area on the detector. In a setup with resolution $R$, the electrons which are longitudinally distributed over the length $R$ do overlap on the screen on an area which is equivalent to the area of the unstreaked beam. Thus, if the same density of electrons on the detector as in the static case ought to be achieved a current of $10^{5} \mathrm{ec} / R$ is required which yields $1.6 \mathrm{~A}$ for $10 \mathrm{fs}$ resolution. While a long emission time mitigates space charge effects near the cathode to some extent it should be noted that electrons pile up in front of the cathode due to their low starting velocity. The total charge in the bunch is hence a relevant quantity for the beam dynamics in the cathode region which limits the current achievable without emittance degradation. Detailed simulation studies will be required to determine the parameter space in which single-shot timeresolved diffraction experiments are competitive to the pump-probe type of experiments.

A diffraction pattern consists of individual spots which can be streaked without losing information by too many overlapping stripes only in case of single crystal diffraction. In case of a polycrystalline or gas target, the diffraction pattern consists however of rings which do overlap when the beam is streaked. To overcome this problem, one can introduce a slit between target and TDS such that only segments of the rings are streaked [3]. The slit should be placed at a position where the diffraction rings are pronounced, i.e., at a focus. The resolution power of the TDS increases however with increasing beam size in the structure; thus, it is desirable to introduce an intermediate focus, at least in the streaking plane, between target and TDS in the case of polycrystalline samples while the TDS can be placed directly behind the target in the case of single crystal diffraction. Besides this complication the loss of signal by the slit has to be noted as a severe drawback of this concept.

\section{AVERAGE TRAJECTORY IN THE TRANSVERSE DEFLECTING STRUCTURE}

Transverse deflecting structures can be realized in the form of traveling wave (TW) or as standing wave (SW) structures. While cylinder symmetric rf modes in accelerating cavities can be described by only three out of the six possible field components, all six components are present in transverse deflecting structures. A standard mode description falls hence short for TDS. A general field description for transverse deflecting structures is discussed in [8]. Important results of the work presented in Ref. [8] will be summarized below; here we start the discussion with a simple model function to point out some general beam dynamics properties.

A transverse deflecting structure should generate a transverse kick which can be expanded to first order as

$$
\begin{aligned}
p_{x} & =\frac{1}{c} \int F_{x} d z \\
& =\frac{e V}{c} \sin \varphi \simeq \frac{e V}{c} \sin \varphi_{0}+\frac{e V}{c} \Delta \varphi \cos \varphi_{0} .
\end{aligned}
$$

$V$ is an effective voltage which is compiled of integrals over electric and magnetic field components. The first term in the expansion yields the average kick that a bunch traversing the structure will receive, while the second term describes the linear correlation that is imprinted onto the bunch.

The standard operating condition for a TDS in diagnostics mode is defined by a phase where the bunch center sees no deflecting field, i.e., $\varphi_{0}=0$. Equation (2) indicates a case where the center of the beam passes on a straight line through the cavity. In a real cavity, however, we can in general not find a phase for which a particle travels unaffected on a straight line, i.e., the particle exits the cavity either with an angle or an offset or a combination of both [9]. Different contributions to this effect can be identified and will be discussed in the following: (i) the interaction with the backward wave and with higher spatial harmonics; (ii) end cell effects; (iii) phase slippage due to velocity mismatch. 
As a reference, the phase where the particle exits without angle is chosen here, so that only an offset has to be considered.

A model for the transverse fields in a standing wave TDS can be written as

$$
E_{x}=E_{x}(z) \cos (\omega t+\varphi), \quad B_{y}=-B_{y}(z) \sin (\omega t+\varphi) .
$$

$E_{x}(z)$ and $B_{y}(z)$ describe the spatial dependence of the transverse field components and can to first order be described by the fundamental spatial harmonics with the wave number $k$ as $E_{x}(z)=E_{0} \sin (k z)$ and $B_{y}(z)=$ $B_{0} \cos (k z) . E_{y}$ and $B_{x}$ are zero. For a particle traveling with matched energy through the cavity $\omega t$ can be replaced by $k z$ so that the force is written as

$$
\begin{aligned}
F_{x}(z)= & e\left(E_{x}-c \beta_{z} B_{y}\right) \\
= & -\frac{e\left(E_{0}-c \beta_{z} B_{0}\right)}{2} \sin \varphi \\
& +\frac{e\left(E_{0}+c \beta_{z} B_{0}\right)}{2} \sin (2 k z+\varphi),
\end{aligned}
$$

with the normalized longitudinal particle velocity $\beta_{z}$.

The effective voltage of the structure follows from the integral of the force over the length $L$ of the cavity as $V=-\left(E_{0}-c \beta_{z} B_{0}\right) \times L / 2 . V$ depends hence on the particle velocity which can - within limits-be compensated by rf power, the velocity dependence of the force due to the second term in Eq. (4) remains however. Because a particle does not "see" the oscillation of a copropagating wave, while the counterpropagating wave is " seen" with twice its frequency, the first term of Eq. (4) can be treated as interaction of the particle with the forward wave, while the second term is interpreted as the interaction with the backward wave. For $\varphi=0$ only the oscillating force of the backward wave contributes to the particle motion. While the average is zero, it still leads to kicks at the entrance and exit of the cavity. The transverse momentum writes as

$$
p_{x}(z)=-\frac{e\left(E_{0}+c \beta_{z} B_{0}\right)}{4 c k}\left[\cos (2 k z)-\cos \left(2 k z_{0}\right)\right],
$$

where the integration constant is introduced such that $p_{x}=0$ at $z=z_{0}$, i.e., at the entrance of the cavity. The integration constant describes an average momentum which the particle receives while traveling through the structure. It is compensated by an equal kick of opposite sign at the cavity exit, so that the trajectory angle is zero upon exit but an offset of

$$
x_{\mathrm{off}}=\frac{e\left(E_{0}+c \beta_{z} B_{0}\right)}{4 c k p_{z}} \cos \left(2 k z_{0}\right) L
$$

remains.
Equation (4) describes the field of a standing wave cavity. In the case of a traveling wave operation, the second term and hence the offset given by Eq. (6) is absent.

The offset can also be influenced by the relative amplitudes of the electric and magnetic components and thus directly by the structure design [10]. In structures with $E_{0}$ and $c B_{0}$ of similar magnitude but opposite sign (often referred to as TM like) $\frac{E_{0}-c B_{0}}{2} \approx E_{0}, \frac{E_{0}+c B_{0}}{2} \approx 0$ holds. The effective backward wave is small in such standing wave structures, while for equal sign (often referred to as TE like) $\frac{E_{0}-c B_{0}}{2}<\frac{E_{0}}{2}$ holds, i.e., the electric and magnetic components counteract each other and a significant backward wave contribution is eminent.

The discussion can be extended to higher spatial harmonics by writing the field components as $E_{x}(z)=$ $\sum E_{p-1} \sin (p k z)$ and $B_{y}(z)=\sum B_{p-1} \cos (p k z), p \geq 1$, which results in

$$
\begin{aligned}
F_{x}(z)= & -e \sum \frac{E_{p-1}-c \beta_{z} B_{p-1}}{2} \sin [\varphi-(p-1) k z] \\
& +e \sum \frac{E_{p-1}+c \beta_{z} B_{p-1}}{2} \sin [(p+1) k z+\varphi] .
\end{aligned}
$$

The effect of the higher harmonics $(p>1)$ is purely oscillatory, i.e., higher spatial harmonics contribute to kicks at the entrance and exit of the cavity in the same way as the backward wave of the fundamental spatial harmonic due to additional sum terms in Eq. (5). A remarkable difference is, however, that the forward wave contribution of the higher spatial harmonics exists also in the case of traveling wave structures. The compensation of electric and magnetic components of the backward wave contribution of the fundamental harmonics as discussed above, reduces however also the total content of higher harmonics [8] and thus reduces both oscillating terms.

The discussion assumes so far a periodic structure. In a real, i.e., finite structure the field leaks however exponentially into the beam pipe which leads to nonperiodic forces in the end cells. In case of a TDS, the situation is complicated as compared to an accelerating structure by the fact that a cosinelike and a sinelike component contribute to the force. The length of the end cells can however be chosen such that the kick of the end cells is minimized or that it even compensates the effect of the backward wave and spatial harmonics [11].

At a few $\mathrm{MeV}$ energies particles travel not with speed of light. The longitudinal velocity is further reduced by the transverse oscillation which a particle performs in the presence of a backward wave or higher spatial harmonics. This leads to a phase shift of the particle relative to the rf wave which has been ignored so far. In an accelerating cavity a small phase shift would effectively only lead to a reduction of the cavity voltage as seen by the particle and 
could be ignored. In a TDS however the phase shift leads to a displacement of the particle trajectory.

Ignoring the oscillating term in Eq. (4), the transverse force acting onto a particle is given as

$$
\begin{aligned}
F_{x} & =-\frac{e\left(E_{0}-c \beta_{z} B_{0}\right)}{2} \sin \left(\varphi_{i}+\frac{\partial \varphi}{\partial z} z\right) \\
& \simeq-\frac{e\left(E_{0}-c \beta_{z} B_{0}\right)}{2}\left(\varphi_{i}+\frac{\partial \varphi}{\partial z} z\right),
\end{aligned}
$$

where $\varphi_{i}$ denotes the initial phase and $\partial \varphi / \partial z$ is the differential phase shift which is assumed to be small. For a structure matched to $\beta_{m}$ and a beam with normalized longitudinal velocity $\beta_{z}, \partial \varphi / \partial z$ is given by

$$
\frac{\partial \varphi}{\partial z}=k_{0}\left(\beta_{m}-\beta_{z}\right)
$$

where $k_{0}$ is the wave number in vacuum. Transverse momentum and offset are hence given by

$$
\begin{aligned}
p_{x} & =\frac{1}{c} \int_{0}^{L} F_{x} d z=-\left.\frac{e\left(E_{0}-c \beta_{z} B_{0}\right)}{2 c}\left(\phi_{i} z+\frac{\partial \phi}{\partial z} \frac{z^{2}}{2}\right)\right|_{0} ^{L}, \\
x_{\text {off }} & =\int_{0}^{L} \frac{p_{x}}{p_{z}} d z=-\frac{e\left(E_{0}-c \beta_{z} B_{0}\right)}{2 c p_{z}}\left(\phi_{i} \frac{L^{2}}{2}+\frac{\partial \phi}{\partial z} \frac{L^{3}}{6}\right) .
\end{aligned}
$$

We choose $\varphi_{i}=-\frac{\partial \varphi}{\partial z} \frac{L}{2}$, so that the transverse momentum $p_{x}$ is zero at the exit of the structure. The transverse offset follows as

$$
x_{\mathrm{off}}=\frac{e V}{12 p_{z} c} \frac{\partial \varphi}{\partial z} L^{2} .
$$

Matching the structure to the velocity of the particles allows one to get rid of this effect but only for a fixed energy as shown in Fig. 1. If some operational flexibility is required,

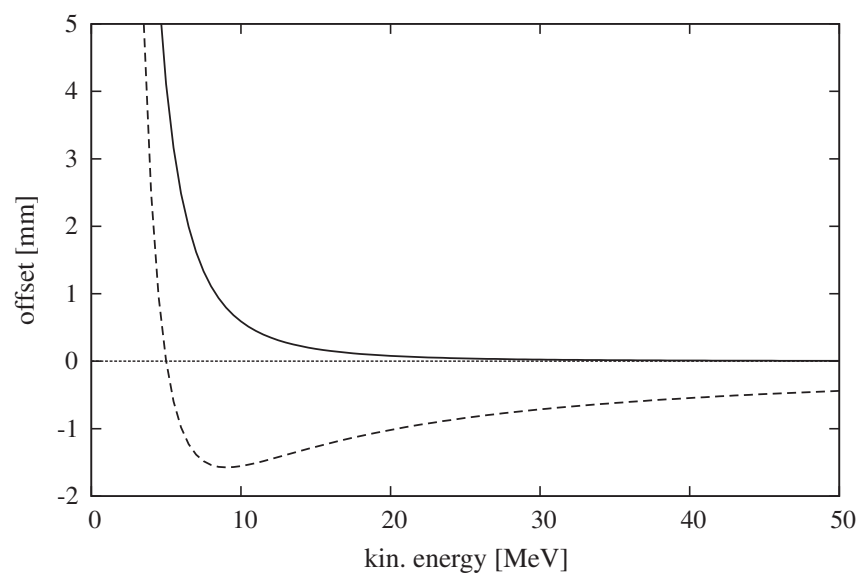

FIG. 1. Transverse offset due to velocity mismatch vs particle energy according to Eq. (11) with $V=1 \mathrm{MV}, L=1 \mathrm{~m}$, $k_{0}=2 \pi / 0.1 \mathrm{~m}^{-1}$. The solid line is for $\beta_{m}=1$, the broken line is matched to $5 \mathrm{MeV}$ particle energy. the matching alleviates the problem but especially for energies below the design energy the offset can still get large. For a larger operating range a short structure is favorable since the offset scales with the square of the structure length. Note that all other contributions discussed above scale with the total deflecting voltage of the cavity but are independent of the length of the structure. Only the contribution due to the beta mismatch can be reduced by a reduction of the structure length and operation at the corresponding higher gradient.

\section{INDUCED ENERGY SPREAD}

A fundamental property of time dependent transverse deflecting fields is that they are accompanied by a longitudinal electric field component, which follows from $\operatorname{rot} \vec{E}=-\frac{\partial}{\partial t} \vec{B}$ as

$$
\frac{\partial}{\partial x} E_{z}=\frac{\partial}{\partial z} E_{x}+\frac{\partial}{\partial t} B_{y}
$$

In the case that the spatial derivative of the electric field and the temporal derivative of the magnetic field add up to zero, the effective transverse voltage $V$ for relativistic particles also becomes zero and thus no deflecting force is generated [12]. A particle traveling through a structure with fields as described in Eq. (3) sees hence a longitudinal field gradient as

$$
\frac{\partial}{\partial x} E_{z}=\frac{k\left(E_{0}-c B_{0}\right)}{2}[\cos \varphi+\cos (2 k z+\varphi)] .
$$

On axis the longitudinal field can be set to zero. The longitudinal voltage follows from the integral over the length of the structure as $V_{z}=-\left(E_{0}-c B_{0}\right) \times L / 2$ and thus $V_{z}=V$ for $\beta_{z}=1$.

The longitudinal field leads to a variation of the mean particle energy in proportion to the average offset of the bunch $\Delta E=-e k V_{z} \cos \varphi_{0} \bar{x}_{\text {off }}$ and an induced uncorrelated energy spread as [1]

$$
\sigma_{E}=e k V_{z} \cos \varphi_{0} \sigma_{x}
$$

where $\sigma_{x}$ is the average rms beam size in the structure.

The energy spread scales with the product of beam size, wave number, and voltage, just as the resolution power $R^{-1}$ [cf. Eq. (1), $V_{z} \simeq V$ ], thus leaving no free parameter for optimization.

To first order no linearly correlated energy spread is induced due to the cosinelike longitudinal electric field operated at $\varphi_{0} \simeq 0$. This changes if the transverse motion of the particles inside the cavity is taken into account. The transverse momentum and position of a particle at phase $\Delta \varphi$ write 


$$
\begin{aligned}
p_{x}(z) & =\int-\frac{e\left(E_{0}-c \beta_{z} B_{0}\right)}{2 c} \cos \varphi_{0} \Delta \varphi d z, \\
x(z) & =\iint-\frac{e\left(E_{0}-c \beta_{z} B_{0}\right)}{2 c p_{z}} \cos \varphi_{0} \Delta \varphi d z^{2} .
\end{aligned}
$$

Only the relative motion inside the bunch needs to be considered at this point [cf. Eq. (2)]; the average motion of the bunch leads to a variation of the mean energy as discussed above. Also the oscillating part of the force can be ignored since the net contribution is zero. With $\Delta \varphi=-k \Delta s$, where $\Delta s$ is the longitudinal position of a particle relative to the bunch center, a linearly correlated energy spread as [1]

$$
\begin{aligned}
\frac{\Delta E^{\mathrm{cor}}}{\Delta s} & =\frac{1}{\Delta s} \int \frac{e k\left(E_{0}-c B_{0}\right)}{2} \cos \phi_{0} x(z) d z \\
& =\frac{(e k V)^{2}}{c p_{z}} \cos ^{2} \phi_{0} \frac{L}{6}, V_{z} \simeq V
\end{aligned}
$$

builds up. The correlated energy spread can in principle be compensated by an accelerating cavity operated at appropriate phase. Moreover gives the dependence on the cavity length some design flexibility, while the uncorrelated energy spread scales just like the resolution power and is in that sense fixed.

The induced energy spread may limit the temporal and transverse resolution due to the chromatic contribution to the beam size on the screen.

\section{BUNCH LENGTHENING AND TRANSVERSE DEFOCUSING}

At beam energies of a few $\mathrm{MeV}$ also the longitudinal motion of the particles within the bunch has to be taken into account.

The longitudinal shift of a particle with a transverse offset $x$ relative to a particle traveling on axis is given as

$$
\Delta z=\int z^{\prime} d z=\int \frac{\Delta \beta(x, z)}{\beta} d z=\int \frac{\Delta E(x, z)}{c p \beta \gamma^{2}} d z,
$$

where $\Delta \beta(x, z)$ and $\Delta E(x, z)$ describe the velocity and energy difference relative to an on-axis particle and $p, \beta$, and $\gamma$ are average momentum, normalized velocity, and relativistic factor of the bunch. Here the relation $\frac{\partial \gamma}{\partial \beta}=\gamma^{3} \beta$ has been used. Thus,

$$
\begin{aligned}
\Delta z(z) & =\iint \frac{e k\left(E_{0}-c B_{0}\right)}{2 c p \beta \gamma^{2}} \cos \varphi_{0} x d z^{2} \\
& =-\frac{e V_{z} k}{c p \beta \gamma^{2}} \cos \varphi_{0} \frac{L}{2} x .
\end{aligned}
$$

The upper part of a bunch (i.e., positive $x$ ) moves hence longitudinally in the opposite direction to the lower part of the bunch and the rms bunch length in the structure develops as

$$
\sigma_{z}^{2}=\sigma_{z 0}^{2}+\left[\frac{e V_{z} k}{c p \beta \gamma^{2}} \cos \varphi_{0} \frac{L}{2} \sigma_{x}\right]^{2},
$$

where $\sigma_{z 0}$ stands for the initial bunch length at the cavity entrance.

For example, the bunch lengthening of a $5.5 \mathrm{MeV} / c$ beam traveling through a $1 \mathrm{~m}$ long S-band structure with $1 \mathrm{MV}$ deflecting voltage $\left(\cos \varphi_{0}=1\right)$ yields $50 \mu \mathrm{m}$ (167 fs) for $\sigma_{x}=1 \mathrm{~mm}$. The bunch lengthening does of course not stop at the end of the cavity but continues due to the induced velocity spread downstream up to the screen.

Due to the bunch lengthening the uncorrelated energy spread of the beam is transferred into a linearly correlated energy spread, so that in principle a correction of the induced energy spread becomes possible. However, the bunch lengthening has not only positive effects, because it perturbs the compensation of correlated emittance oscillations inside the cavity as will be discussed below. In addition it leads to a transverse defocusing in the streaking direction which disturbs the linear beam optics.

Writing the transverse momentum $p_{x}$ of a particle with a longitudinal distance $\Delta \varphi=-k \Delta s$ relative to the bunch center as

$$
p_{x}=\int \frac{e k\left(E_{0}-c \beta_{z} B_{0}\right)}{2 c} \cos \varphi_{0} \Delta s d z,
$$

we find by introducing the bunch lengthening [Eq. (18)],

$$
\begin{aligned}
p_{x} & =\int \frac{e k\left(E_{0}-c \beta_{z} B_{0}\right)}{2 c} \cos \varphi_{0}\left[\Delta s_{0}+\Delta z(z)\right] d z \\
& =\frac{e V k}{c} \cos \varphi_{0}\left[-\Delta s_{0}+\frac{e V_{z} k}{c p \beta \gamma^{2}} \cos \varphi_{0} \frac{L}{6} x\right],
\end{aligned}
$$

where $\Delta s_{0}$ stands for the initial longitudinal position of the particle relative to the bunch center.

The transverse momentum receives hence an additional component which depends on the transverse position of the particle, i.e., the bunch is defocused in the streaking direction. Figure 2 compares the development of the transverse momentum of a single particle with and without consideration of the bunch lengthening in a $1 \mathrm{~m}$ long structure. A compensation of the defocusing action with an appropriate optics is conceivable but tuning may become difficult in praxis. More studies are required to conclude on this; anyhow it is preferable to minimize this effect as far as possible to avoid complications.

The ratio of the two terms in the bracket describes the relative contribution of the defocusing effect onto the beam size if no correction is applied. Introducing the shear parameter $S$ (assuming $V_{z}=V$ ) yields with $\cos \varphi_{0}=1$ and $p=p_{z}$ : 


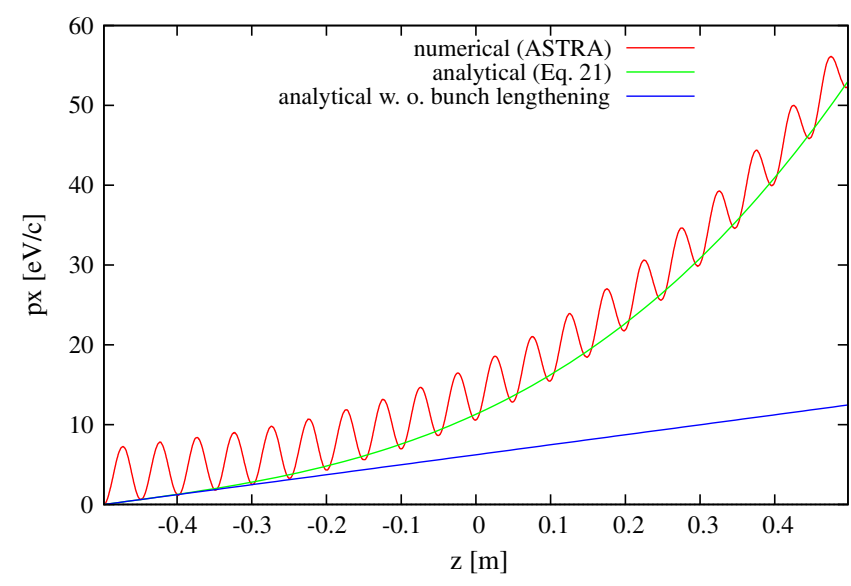

FIG. 2 (color online). Development of the transverse momentum in a $1 \mathrm{~m}$ long $\mathrm{S}$-band structure $\left(\cos \varphi_{0}=1\right)$. The particle starts parallel to the cavity axis with $1 \mathrm{~mm}$ transverse offset and $1 \mu \mathrm{m}$ longitudinal offset relative to the bunch center. The structure is matched to the particle energy of $5 \mathrm{MeV}$ and has an effective voltage of $-0.2 \mathrm{MV}$.

$$
\frac{p^{\text {def }}}{p^{\text {shear }}}=\frac{S}{\beta \gamma^{2}} \frac{L}{6} \frac{x}{\Delta s_{0}} .
$$

The length scale which we want to observe is given by the resolution, thus we may replace $\Delta s_{0}$ by $R$ furthermore $x$ by $\sigma_{x}$ and $S \sigma_{x}$ by $\varepsilon / R$ to get

$$
\frac{p^{\mathrm{def}}}{p^{\text {shear }}}=\frac{\varepsilon}{R^{2} \beta \gamma^{2}} \frac{L}{6}
$$

i.e., the finer the resolution the more sensitive is the measurement to defocusing. Due to the $\gamma^{-3}$ scaling (the geometrical emittance scales with $\gamma^{-1}$ ) defocusing is effective only at low energies. Shorter structures are preferable since they generate less defocusing than longer structures.

\section{INTERMEDIATE DISCUSSION}

While the induced uncorrelated energy spread [Eq. (14)] scales as the resolution power and thus cannot be influenced by the cavity design, other effects like the offset introduced by a velocity mismatch [Eq. (11)], the correlated energy spread [Eq. (16)], the bunch lengthening [Eq. (19)], and also the defocusing [Eq. (21)] scale explicitly with the structure length. Shorter structures are hence preferable to keep these effects small.

For a fixed deflecting voltage, shorter structures are less efficient in terms of $\mathrm{rf}$ power required to build up the field because power losses scale quadratically with the gradient. Especially problematic are traveling wave structures in which the rf wave is propagating through the cavity while being attenuated due to power dissipation in the cavity walls. At the end of the structure the remaining power needs to be dumped (internally or externally) rather than being reflected as in the case of a standing wave cavity. A high rf efficiency requires that only a small fraction of the energy is dumped which is difficult to realize in the case of short structures. For a detailed discussion on the dependence of the rf efficiency on the cavity parameters, see Ref. [8].

According to Eq. (1) a good resolution can also be achieved with a low deflecting voltage but a large beam size in the structure. Ignoring all practical difficulties related to an operation at unusual large beam size, this approach finds its limits in the nonlinearities of the deflecting field itself as will be discussed in the following. Concerning the TDS design we are hence seeking for a short structure with high rf efficiency and linear deflecting field.

\section{ANALYSIS OF DEFLECTING FIELDS}

For the description of deflecting fields hybrid $H E$ and $H M$ waves were introduced $[13,14]$ in which the longitudinal components $E_{z}$ and $B_{z}$ are dependent on each other and are not vanishing if the period length is matched for $\beta_{z} \rightarrow 1$. This is necessary because the more common description in terms of $T E$ and $T M$ waves leads to a degenerated TEM wave with no transverse deflection for $\beta_{z} \rightarrow 1$ matching.

A general solution of the fields in the aperture of a transverse deflecting structure can be given in the form of a superposition of the hybrid waves [13] like

$$
\vec{E}=a \vec{E}_{H E_{1}}+b \vec{E}_{H M_{1}}
$$

where the coefficients $a$ and $b$ describe the relative contribution of the basis waves which is determined by the geometry of the supporting structure.

For the disk loaded waveguide (DLW) structure, the well-known field distribution was obtained in the small pitch approximation [14], which neglects all higher spatial harmonics:

$$
\begin{aligned}
E_{x}(z) & =\frac{\hat{E}}{8} k^{2}\left[r_{a}^{2}+x^{2}-y^{2}\right] \sin (k z), \\
c B_{y}(z) & =\frac{\hat{E}}{8} k^{2}\left[r_{a}^{2}+x^{2}-y^{2}-\frac{4}{k^{2}}\right] \cos (k z), \\
E_{y}(z) & =\frac{\hat{E}}{4} k^{2} x y \sin (k z), \quad c B_{x}(z)=-\frac{\hat{E}}{4} k^{2} x y \cos (k z), \\
E_{z}(z) & =\frac{\hat{E}}{2} k x \sin (k z), \quad c B_{z}(z)=-\frac{\hat{E}}{2} k y \cos (k z),
\end{aligned}
$$

where $r_{a}$ denotes the radius of the iris aperture.

The approximation requires matched structures $\beta_{m}=1$ and that the structure cell length $d$ is shorter than the wavelength $\lambda, d \ll \lambda$, and the iris thickness $t$ is smaller than the cell length, $t \ll d$. 
Both the electric and the magnetic field are nonlinear. The nonlinearities cancel in the deflecting force $F_{x}=e\left(E_{x}-c \beta_{z} B_{y}\right)$ only for relativistic particle energies $\beta_{z}=1$. Moreover, an oscillating term shows up in the standing wave case in the streaking direction but also in the direction perpendicular to the streaking direction. Thus, only for $\beta_{z}=1$ and in traveling wave mode,

$$
F_{x}(z)=\frac{e \hat{E}}{2}=\text { const, } \quad F_{y}(z)=0,
$$

is reached. The application of the small pitch approximation for disk loaded waveguides operating both in standing wave and traveling wave mode is considered in more detail in Ref. [8].

The general solution of deflecting fields reveals additional, nonlinear components. In the following the expansion of the field near the axis is employed, because from the beam dynamics point of view only the dependence of the field components near the axis is relevant.

The first addition arises only in cavities with a period length matched to the particle velocity $\beta_{z} \neq 1$. A simple expansion for the distribution of the fundamental harmonics leads to a description as

$$
\begin{aligned}
F_{x}(z) & \approx \frac{e\left(a_{0} k_{z 0}-b_{0} k\right)}{2 k_{z 0}}\left(1+k_{s 0}^{2} \frac{x^{2}+y^{2}}{4}+\cdots\right), \\
F_{y}(z) & \approx \frac{e\left(a_{0} k_{z 0}-b_{0} k\right)}{2 k_{z 0}}\left[k_{s 0}^{4}\left(x^{3} y+y^{3} x\right)+\cdots\right], \\
k & =\frac{\omega}{c}, \quad k_{z 0}=\frac{k}{\beta_{z}}, \quad k_{s 0}^{2}=\left|k^{2}-k_{z 0}^{2}\right| .
\end{aligned}
$$

In the nonrelativistic case already the field of the synchronous harmonics is not free of nonlinear additions. The aberrations vanish with $\frac{1}{\beta_{3}^{2} \gamma^{2}}$ for higher energies.

The second addition is the main source for transverse nonlinearities and arises from higher spatial harmonics. The effective deflecting force of the $p$ th harmonic can be written as

$$
\begin{aligned}
& F_{x p} \approx \frac{e k_{s p}\left(a_{p}+b_{p}\right)}{2}\left(1+k_{s p}^{2} \frac{x^{2}+y^{2}}{4}+\cdots\right) e_{z p}, \\
& F_{y p} \approx \frac{e k_{s p}\left(a_{p}+b_{p}\right)}{2}\left[k_{s p}^{4}\left(x^{3} y+y^{3} x\right)+\cdots\right] e_{z p}, \\
& k_{z p}=\frac{k\left(\theta_{0}+2 \pi p\right)}{\beta_{z} \theta_{0}}, \quad k_{s p}^{2}=\left|k_{z p}^{2}-k^{2}\right|,
\end{aligned}
$$

where $\theta_{0}$ is the operating phase advance of the TDS and $e_{z p}$ is the corresponding longitudinal field component of the $p$ th harmonic. Equation (28) shows a great formal similarity to Eq. (27). However, the contribution of the $p$ th harmonic vanishes only if $a_{p} \simeq-b_{p}$ while the nonlinear addition to the synchronous mode vanishes only if the structure is matched to $\beta_{z}=1$.
Another remarkable point is that for opposite phasing of the hybrid waves $H E$ and $H M$, i.e., $a \times b<0$ in Eq. (24), the higher spatial harmonic of the electric field partially compensates the corresponding harmonic of the magnetic field, while for equal phasing $a \times b>0$ the amplitude of the deflecting force of the $p$ th harmonic is even larger than the amplitudes of the corresponding field components. Transverse deflecting structures with a pronounced predominance of the transverse electric field in the aperture $\left|E_{x}\right| \gg\left|E_{z}\right|$ ensue an equal phasing of the hybrid waves.

Spatial harmonics in the electromagnetic field components are essential at the TDS aperture $r=r_{a}$, but attenuate toward the axis as

$$
e_{p}(0) \sim e_{p}\left(r_{a}\right) \exp \left(-\frac{4 \pi^{2} p}{\beta \theta_{0}} \frac{r_{a}}{\lambda}\right), \quad p>1 .
$$

Nonlinear additions in the deflecting force are hence reduced in TDS with large apertures, operating in traveling wave mode with low phase advance $\theta_{0} \ll \pi$.

The third addition arises due to a break of the rotational symmetry of the cavity structure which is either a feature of the original TDS design or which needs to be introduced in order to define the direction of the deflecting field. The strongest component has a sextupole wave structure. With the amplitude $\hat{E}_{s}$ the force in the transverse directions even of the fundamental harmonic writes as

$$
F_{x}=\frac{e \hat{E}_{s}}{16}\left[k^{2}\left(x^{2}-y^{2}\right)\right], \quad F_{y}=\frac{e \hat{E}_{s}}{16} 2 k^{2} x y .
$$

For the simple disk loaded waveguide structure in standing wave mode, the most efficient reduction of higher harmonics [Eq. (28)] is achieved [10] by enlarging the iris aperture to balance the electric and magnetic field components so that $a \simeq-b$. At the same time the effect of the backward wave onto the average trajectory [Eq. (5)] is reduced. It leads however also to a reduction of the $\mathrm{rf}$ efficiency. Balancing the field components while keeping a high rf efficiency requires to decouple the control of the TDS rf parameters and of the field distribution near the axis [15] which requires structure designs with more degrees of freedom than the classical disk loaded waveguide.

Estimates of the harmonic content in deflecting structures and following design criteria for structures with minimized nonlinear additions are discussed in detail in Ref. [8] and the references therein.

The emittance is the product of transverse momentum and transverse size, thus a force scaling with the transverse dimension to the power of $n$ yields an emittance scaling with the power of $n+1$. Assuming a round beam we can thus expect from the above equations (27) and (28) an emittance growth in the streaking direction which scales linearly with the transverse beam size - this is the desired action of the streaking force and calculates as 
$\epsilon_{n, x}^{\operatorname{lin}}=\frac{e V k}{m_{0} c^{2}} \sigma_{z} \sigma_{x}$-plus a nonlinear term scaling with the third power of the transverse beam size. Both terms add in quadrature. In the direction perpendicular to the streak both equations yield a scaling with the power of 4 .

The nonlinear additions lead also to an emittance growth in dependence on the average transverse position of the bunch in the structure. Keeping the average trajectory on the structure axis as discussed above as well as a good alignment of the structure is hence mandatory.

\section{SIMULATION OF EMITTANCE GROWTH}

The discussion above provides guidelines for the design of a TDS for $\mathrm{MeV}$ particle energies. To confirm the general conclusions and select the most promising TDS option we investigated a large parameter space of structure designs in the $S$-band frequency range $(\lambda=0.1 \mathrm{~m})$ both in terms of $\mathrm{rf}$ parameters and in terms of the resulting beam dynamics. In order do this efficiently, we tracked a bunch of particles through single cells only. The results cannot be directly extrapolated to a complete cavity design but allow for a fast and direct comparison of structures. A selection of the investigated cell geometries is shown in Fig. 3, together with the electric field distribution, calculated with Microwave Studio [16]. More structure parameters are summarized in Table I, while Table II provides initial parameters of the particle distribution which was tracked through the cavities with the program ASTRA [17].

Structures A and B in Fig. 3 have a very high rf efficiency, especially structure B with the small aperture radius $r_{a}=6.72 \mathrm{~mm}$, as the rf efficiency decreases with increasing aperture radius [18]. Structure A can be considered either as an evolution of structure B [18] or as an evolution of a parallel-bar structure [19]. Both structures, $\mathrm{A}$ and $\mathrm{B}$, ensue an equal phasing of the hybrid waves $H E$,

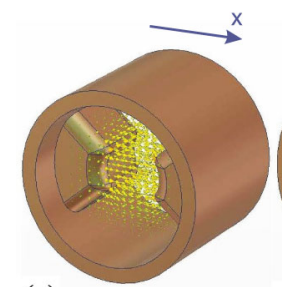

(a)

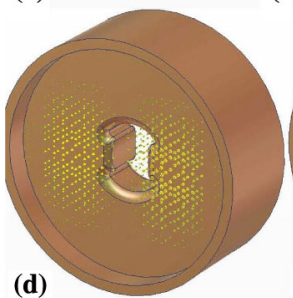

(b)
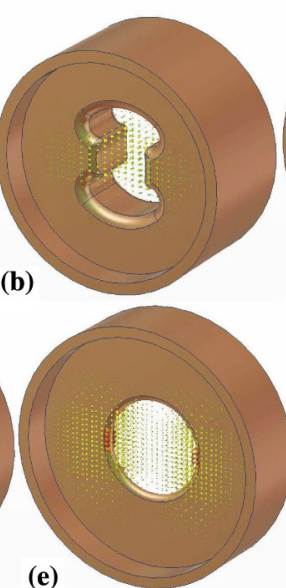

(c)

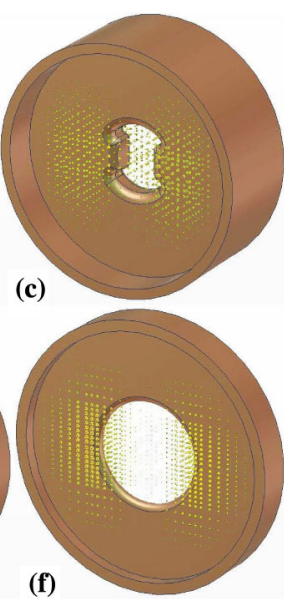

FIG. 3 (color online). Cell geometries and distribution of the electric field in different TDS options. The arrow shows the direction of the deflection $x$.
TABLE I. Main parameters of the example structures; $\theta_{0}=$ phase advance, $\beta_{g}$ group velocity for traveling wave operation, $r_{a}=$ minimal aperture radius, $Q=$ unloaded quality factor, $Z_{e}=$ shunt impedance.

\begin{tabular}{lcccccc}
\hline \hline Structure & $\begin{array}{c}\text { Operating } \\
\text { mode }\end{array}$ & $\theta_{0}$ & $\beta_{g}$ & $r_{a} \mathrm{~mm}$ & $Q 10^{3}$ & $Z_{e} \frac{\mathrm{M} \Omega}{\mathrm{m}}$ \\
\hline $\mathrm{A}$ & $\mathrm{SW}$ & $\pi$ & & 10.0 & 8.35 & 48.4 \\
$\mathrm{~B}$ & $\mathrm{SW}$ & $\pi$ & & 6.72 & 9.38 & 61.0 \\
$\mathrm{C}$ & $\mathrm{SW}$ & $\pi$ & & 10.0 & 13.8 & 36.7 \\
$\mathrm{D}$ & $\mathrm{SW}$ & $\pi$ & & 10.0 & 12.35 & 32.0 \\
$\mathrm{E}$ & $\mathrm{TW}$ & $\frac{2 \pi}{3}$ & -0.01 & 22.3 & 12.3 & 16.8 \\
$\mathrm{~F}$ & $\mathrm{TW}$ & $\frac{\pi}{3}$ & -0.02 & 23.8 & 8.0 & 12.2 \\
\hline \hline
\end{tabular}

$H M$ and, in accordance with Eqs. (5) and (28), obey a strong backward wave and nonlinear fields.

Structure E repeats the classical LOLATDS [20]—a disk loaded waveguide structure in traveling wave mode with phase advance $\theta_{0}=\frac{2 \pi}{3}$. As compared to option E, structure F has-due to the reduced phase advance and a better field balance-a roughly 6 times reduced level of aberrations of the deflecting field and an about 40 times lower aberration level of the longitudinal field. Structures E and F have no multipole additions in the field distribution and according to the field simulations [8] structure $\mathrm{F}$ has the minimal level of aberrations of all structures inside the investigated parameter space. The rf efficiency of option $\mathrm{F}$ is however only moderate.

Structures C and D form a compromise in terms of if efficiency and field nonlinearity. The effect of the backward wave [cf. Eq. (5)] is in option $\mathrm{C}$ overall-i.e., both from the fundamental and from higher spatial harmonicsminimized by realizing the concept of a decoupled parameter control [18]. It results in a balance of the field components of the fundamental harmonics [cf. Eq. (4)] as $0.8 E_{0} \approx c B_{0}$. For structure $\mathrm{D}$ a precise balancing is realized as $1.0008 E_{0}=c B_{0}$ [10] and oscillating force terms exist due to higher spatial harmonics only.

Below we concentrate the discussion on the emittance growth in the three example structures $\mathrm{B}, \mathrm{C}$, and F.

The initial bunch has zero emittance, so that emittance growth can be easily studied and it starts on axis. Except for structure B the fields are compensated such that the beam stays in close vicinity to the cavity axis while traveling through the structure. The streaking voltage is set to $1 \mathrm{MV}$ and the phase is adjusted to the nominal zero as defined

TABLE II. Important initial parameters of the bunch for the tracking calculations.

Transverse distribution Round, top-hat, varying diameter

Longitudinal distribution Uniform, rms length $\sigma_{z 0}=10^{-4} \mathrm{~mm}$

Energy spread

Transverse

$\sigma_{E}=0.0$

Emittance

$\epsilon_{x}=0.0$

$\epsilon_{y}=0.0$ 

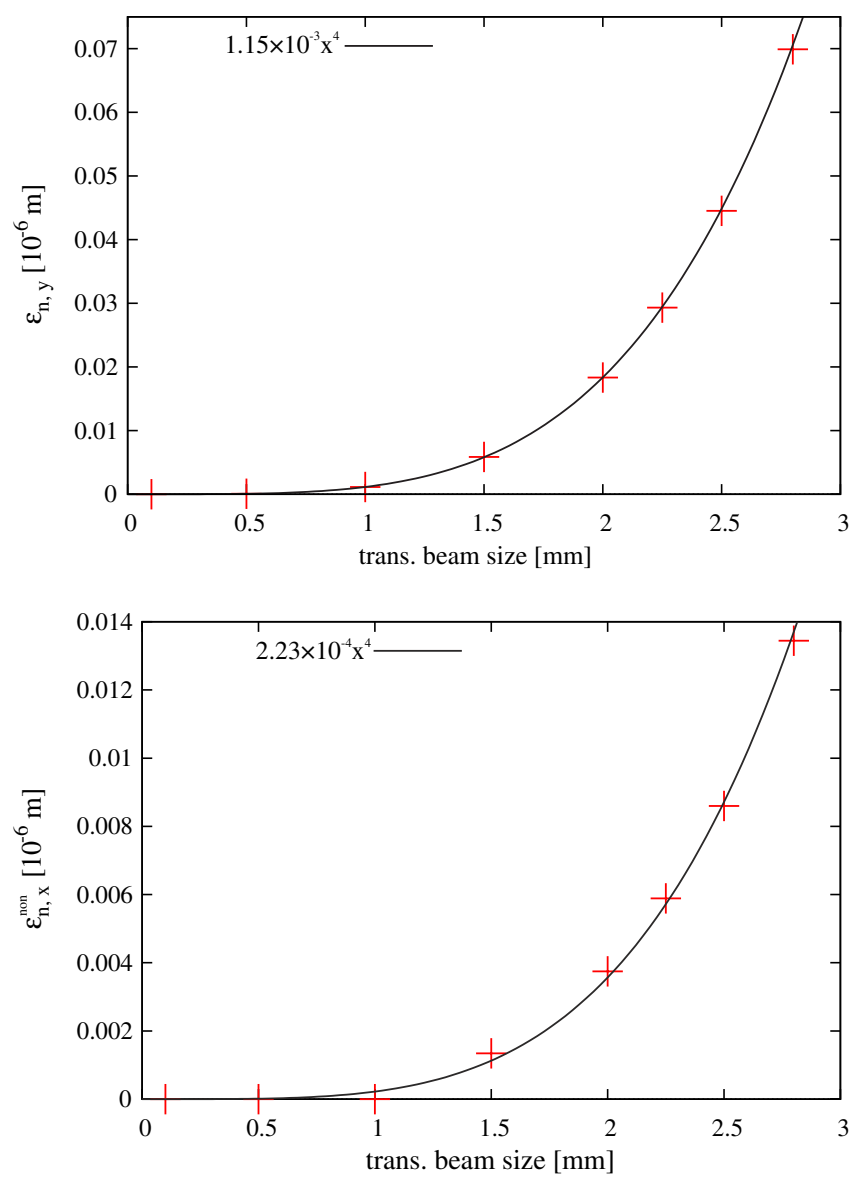

FIG. 4 (color online). Normalized emittance in a direction perpendicular to the streak vs beam size (top) and nonlinear normalized emittance in the streaking direction vs beam size for the compensated structure $\mathrm{C}$ at a beam energy of $50 \mathrm{MeV}$ and power of 4 fit. Beam parameters are according to Table II.

above. Figure 4 (top) shows the normalized emittance growth in the direction perpendicular to the streak for the decoupled structure $\mathrm{C}$ vs the transverse beam size together with a power of 4 fit. Here a beam with $50 \mathrm{MeV}$ particle energy was used, to avoid bunch lengthening and corresponding second order effects. The nonlinear addition in the streaking direction is covered by the linear emittance growth introduced due to the streaking action. Thus, in Fig. 4 (bottom) the emittance after quadratic subtraction of the theoretical linear part is shown together with a fit. In contrast to the expectation the emittance scales rather with the fourth power of the beam size than with the third power.
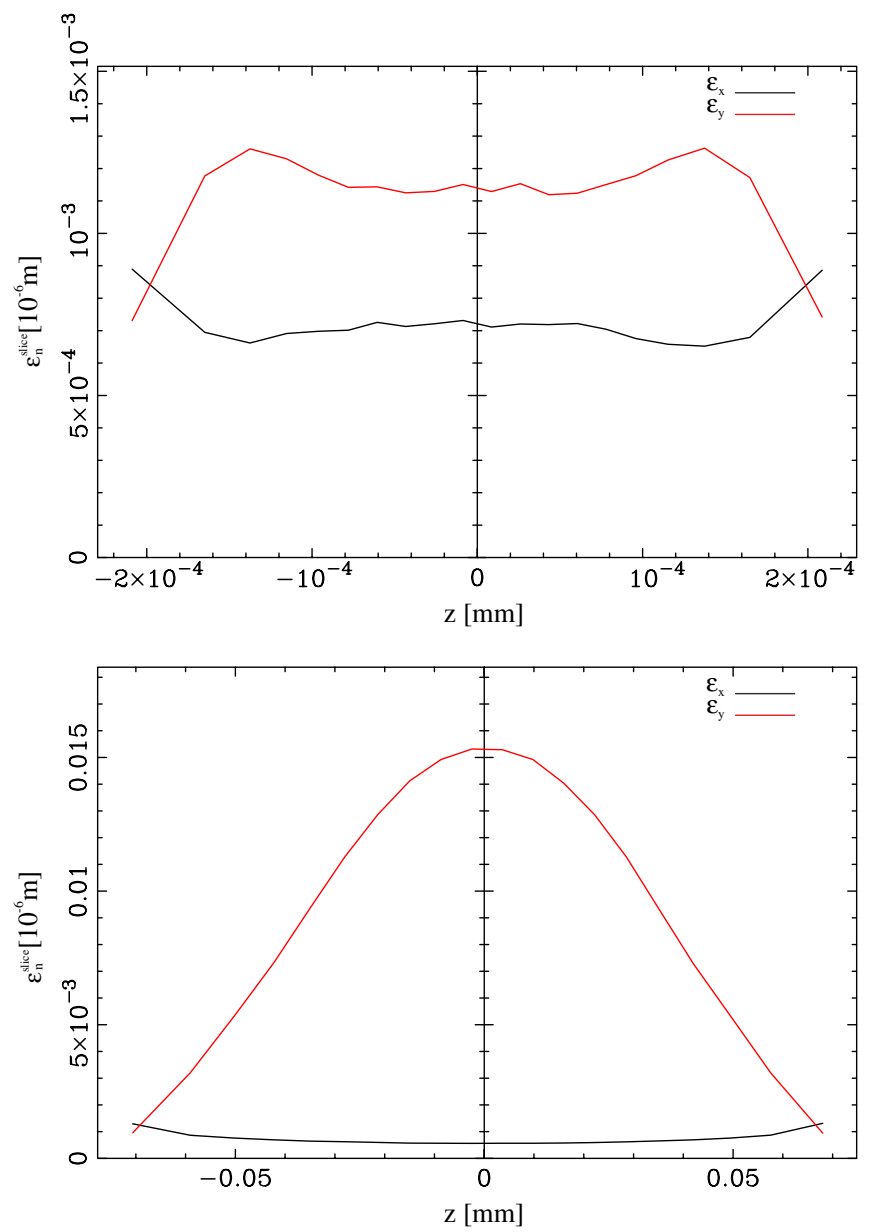

FIG. 5 (color online). Comparison of the slice emittance at $50 \mathrm{MeV}$ (top) and at $5 \mathrm{MeV}$ (bottom) for structure C.

Also for the other structures which show a larger emittance growth the data do not always agree to the expected scaling. Structure $F$ for example gives better fits for a power of 4 law in the streaking direction and a power of 3 law in the perpendicular direction. These discrepancies are likely related to numerical inaccuracies and need more study.

Table III compares the emittance growth at $50 \mathrm{MeV}$ particle energy with the emittance growth at $5 \mathrm{MeV}$ energy at $1 \mathrm{~mm}$ rms beam size. As in Fig. 4 (bottom) the linear emittance growth in the streaking direction has been quadratically subtracted, i.e., only the nonlinear addition is shown.

TABLE III. Comparison of induced emittance growth at $50 \mathrm{MeV}$ and at $5 \mathrm{MeV}$ beam energy. The rms beam size is $1 \mathrm{~mm}$; in the streaking $x$ direction the linear emittance contribution has been quadratically subtracted.

\begin{tabular}{lcccc}
\hline \hline Structure & $\epsilon_{n, x}^{\text {non }} 10^{-6} \mathrm{~m}$ at $50 \mathrm{MeV}$ & $\epsilon_{n, x}^{\text {non }} 10^{-6} \mathrm{~m}$ at $5 \mathrm{MeV}$ & $\epsilon_{n, y} 10^{-6} \mathrm{~m}$ at $50 \mathrm{MeV}$ & $\epsilon_{n, y} 10^{-6} \mathrm{~m}$ at $5 \mathrm{MeV}$ \\
\hline $\mathrm{B}$ & $8.1 \times 10^{-2}$ & 0.68 & $3.4 \times 10^{-2}$ & 0.69 \\
$\mathrm{C}$ & 0.0 & $3.9 \times 10^{-3}$ & $1.1 \times 10^{-3}$ & $1.0 \times 10^{-2}$ \\
$\mathrm{~F}$ & $3.5 \times 10^{-3}$ & $4.0 \times 10^{-3}$ & $3.7 \times 10^{-3}$ & $3.7 \times 10^{-3}$ \\
\hline \hline
\end{tabular}


The decoupled structure $\mathrm{C}$ yields at $50 \mathrm{MeV}$ even slightly better results than the disk loaded wave guide structure.

At $5 \mathrm{MeV}$ a strong increase of the induced emittance especially perpendicular to the streaking direction is observed in the standing wave structures. The origin of this emittance contribution is found in correlated emittance oscillations introduced by oscillating field terms primarily in the standing wave structures. The bunch lengthening disturbs the internal compensation of these oscillations at lower particle energies. This becomes also apparent in the slice emittance at the two energies which is compared in Fig. 5 for structure C. In the streaking direction the slice emittance is flat and increases only slightly toward the head and tail while it increases strongly in the center of the bunch in the perpendicular direction. The emittance growth is driven by the emittance oscillation and the longitudinal motion per cell, which both scale with the streaking gradient but it is additive from cell to cell. The scaling for a complete structure is hence found to be proportional to $V^{2} / L$. This effect calls thus for longer structures.

\section{FINAL CAVITY DESIGN}

Based on structure $\mathrm{C}$, a complete cavity with matched end cells (i.e., the beam stays on axis) has been designed, [10]. Figure 6 shows the structure consisting of four regular-including the RF coupler cell—and two end cells. The structure is beta matched to a beam energy of $5 \mathrm{MeV}$. As compared to the single cell results, the emittance growth is reduced by a factor 2 for the projected and the slice emittance in the $y$ direction at $5 \mathrm{MeV}\left(\varepsilon_{n, y}=4.7 \times 10^{-9} \mathrm{~m}\right.$ projected; $\varepsilon_{n, y}=7.1 \times 10^{-9} \mathrm{~m}$ central slice emittance).

The structure has a calculated shunt impedance of $6.04 \mathrm{M} \Omega$. It is foreseen to drive it with a $5 \mathrm{~kW}$ rf power amplifier which allows for a maximal deflecting voltage of $170 \mathrm{kV}$. For this low rf power a simple loop antenna may be employed as coupler. Assuming a normalized emittance of

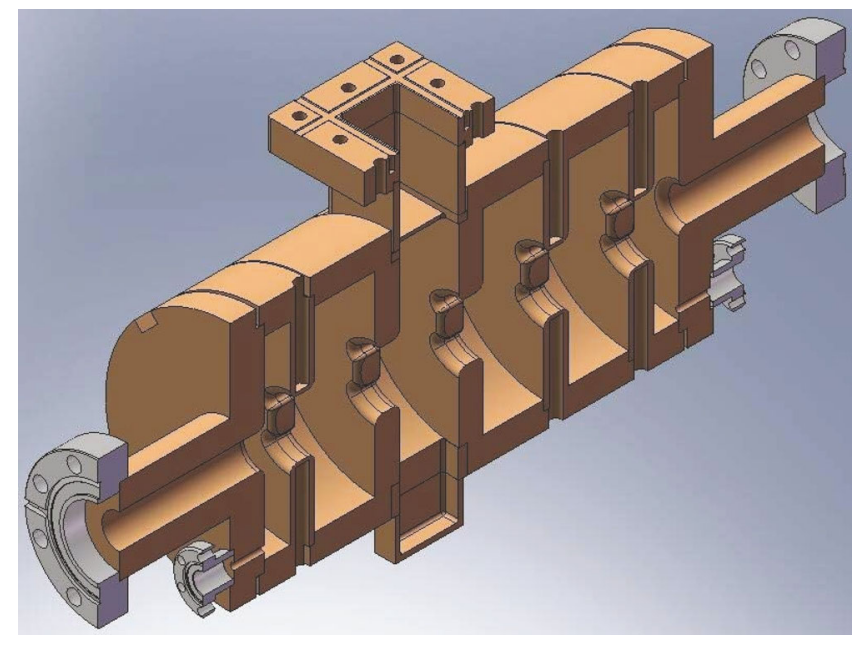

FIG. 6 (color online). Final cavity design with coupler and end cells.
$10 \mathrm{~nm}$ and a beam size of $0.4 \mathrm{~mm}$ in the structure, this yields a theoretical resolution of 4.0 fs. The defocusing contribution [cf. Eq. (23)] yields a $20 \%$ contribution to the beam size. Due to the lower voltage and the smaller beam size (as compared to the reference values used above) the emittance growth is negligible. In praxis the resolution will be further influenced by misalignments, field imperfections, phasing errors, etc.; still a resolution of about $10 \mathrm{fs}$ appears to be feasible. This promising value relies on a small emittance as required anyhow for high quality diffraction experiments. Measurements of beams with larger emittances require stronger deflecting voltages (and/or a larger beam size in the structure). A compensation of the defocusing effect will become necessary in this case.

\section{SUMMARY}

The performance of transverse deflecting structures operated in streaking mode is limited by the beam dynamics in the structures as well as by the available rf power and the rf efficiency, respectively.

Transverse offsets of the average particle position are related to end cell effects, to the interaction with the backward wave, and to phase slippage due to a velocity mismatch. At slightly nonrelativistic energies bunch lengthening and transverse defocusing are important effects which need to be taken into account.

Special attention has to be paid to the linearity of the rf fields if high quality beams are required. A detailed analysis of the field components reveals the origin of nonlinear contributions and yields a basis for an effective suppression of the nonlinearities in combination with an efficient rf design.

\section{ACKNOWLEDGMENTS}

The study of deflecting field structures was supported in part by Grant No. RBFR N12-02-00654-a.

[1] P. Emma, J. Frisch, and P. Krejcik, SLAC Report No. LCLS-TN-00-12, 2000.

[2] M. Roehrs, Ch. Gerth, H. Schlarb, B. Schmidt, and P. Schmueser, Phys. Rev. ST Accel. Beams 12, 050704 (2009).

[3] G. Mourou and S. Williamson, Appl. Phys. Lett. 41, 44 (1982).

[4] R. Li, W. Huang, Y. Du, J. Shi, and Ch. Tang, Nucl. Instrum. Methods Phys. Res., Sect. A 637, S15 (2011).

[5] REGAE [http://regae.desy.de/].

[6] R. Li, G. Roberts, C. M. Scoby, H. To, and P. Musumeci, Phys. Rev. ST Accel. Beams 15, 090702 (2012).

[7] R. Li, P. Musumeci, H. A. Bender, N. S. Wilcox, and M. Wu, J. Appl. Phys. 110, 074512 (2011).

[8] V. V. Paramonov, arXiv:1302.5306v1.

[9] D. Edwards, "Note on transit on deflecting mode pill box cavity," Fermilab Note, 2007. 
[10] V. Paramonov, K. Floettmann, L. Kravchuk, and P. Orlov, Proceedings of RuPAC, 2012, Saint Petersburg, 2012.

[11] K. J. Kim, Report No. ANL-AAI-TN-2007-01, 2007; J. Shi, H. Chen, Ch. Tang, S. Zheng, W. Huang, J. Power, Ch. Jing, K. J. Kim, W. Gai, and D. Li, Nucl. Instrum. Methods Phys. Res., Sect. A 598, 388 (2009).

[12] W. K. H. Panofsky and W. A. Wenzel, Rev. Sci. Instrum. 27, 967 (1956).

[13] H. Hahn, Rev. Sci. Instrum. 34, 1094 (1963).

[14] Y. Garault, Cern Report No. 64-43, 1964.
[15] V. Paramonov, Proceedings of Linac 2012, Tel Aviv, 2012.

[16] Microwave Studio, CST GmbH, Darmstadt, Germany [http://www.cst.com].

[17] K. Floettmann, ASTRA-A space charge tracking algorithm [http://www.desy.de/ mpyflo].

[18] V. Paramonov, L. Kravchuk, and K. Floettmann, Proceedings of Linac 2012, Tel Aviv, 2012.

[19] S. U. De Silva and J. R. Delayen, Phys. Rev. ST Accel. Beams 16, 012004 (2013).

[20] O. H. Altenmueller, R. R. Larsen, and G. A. Loew, Rev. Sci. Instrum. 35, 438 (1964). 